\section{Medizinische Fachpresse und Publikumspresse Medienpreis Bipolar 2003}

\section{$\mathrm{V}$} er Beiträge aus den Kategorien medizinische Fachpresse und Publikumspresse wurden am 13. Mai 2004 in Frankfurt am Main mit dem „Medienpreis Bipolar 2003“ der Lilly Deutschland GmbH ausgezeichnet. Der Preis steht unter der

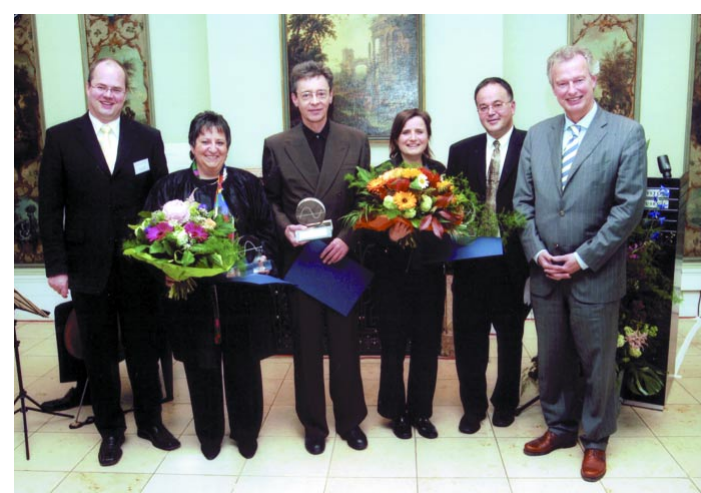

Medienpreis Bipolar: von links nach rechts: Dr. Gert Kräh, Lilly Deutschland GmbH, Christine Vetter, Dr. Eberhard Wormer, Dr. Ulrike Schäfer, Dr. Marius Houchangnia, Mitglied der Jury, Klaus Peter Siegloch
Schirmherrschaft der Deutschen Gesellschaft für Bipolare Störungen e.V. (DGBS). Die beiden Hauptpreisträger erhalten für ihre ausgezeichneten Arbeiten je 2.600 Euro.

Mit dem Preis werden journalistisches Engagement und verantwortungsbewusste Rechercheprojekte auf dem Gebiet der bipolaren Erkrankung gewürdigt. Der „Medienpreis Bipolar 2003“ wurde im Rahmen eines Festakts im Frankfurter Goethehaus durch den Fernsehjournalisten Klaus-Peter Siegloch überreicht.

Die freie Medizinjournalistin Christine Vetter erhielt den „Medienpreis Bipolar 2003“ in der Kategorie medizinische Fachpresse für ihren Artikel „Bipolare Störungen manisch depressiv“, der in der Zeitschrift ZM Zahnärztliche Mitteilungen erschienen ist.

Dr. med. Eberhard J. Wormer ist ebenfalls freier Medizinjournalist und wurde als Ehrenpreisträger in der Kategorie medizinische Fachpresse für seine kommentierte Online-Edition „Bipolar-Klassiker im Internet - Kraepelin online - Das manisch-depressive Irresein“ ausgezeichnet.

In der Kategorie Publikumspresse (Print, TV, Hörfunk) vergab die Jury den Preis an Dr. med. Ulrike Schäfer für das Buch „Im Auf und Ab der Gefühle“. Der Ratgeber für Angehörige und Betroffene, der in $\mathrm{Zu}$ sammenarbeit mit Professor Dr. med. Eckart Rüther entstanden ist, ist im Wissenschaftsverlag erschienen.

Die Journalistin Anke Nolte wurde für ihren Artikel „Zwischen Himmel und Hölle“, Bonner General Anzeiger, mit dem Ehrenpreis in der Kategorie Publikumspresse ausgezeichnet.

Nach Auffassung der unabhängigen Experten-Jury haben die Preisträger mit ihren Projekten einen außerordentlichen Beitrag zur intensiven Aufklärung über das häufig vorkommende, aber selten erkannte Krankheitsbild geleistet.

Weitere Informationen unter http://dgbs.de/presse/index.php 